\title{
Warmes Blut für massive Transfusion
}

\section{Leitartikel}

Wenn ein Patient grosse Mengen gelagerten Blutes innerhalb kurzer Zeit erhalten muss, zum Beispiel in der Gefässchirurgie, bei multiplen Verlet-zungen oder radikalen Krebsoperationen, so kann die rasche Transfusion

1 Originaltitel: Warm blood for massive transfusion.

\section{6}

Warmes Blut für massive Transfusion

kompatiblen Blutes an sich gefährlich sein, da dadurch ein Zirkulations-stop eintreten kann. Blutkonserven mit Zitrat-Glukose-Medien bedingen durch den Zitratüberschuss eine Depression des ionisierten Kalziums, ein Umstand, der eine Zirkulationsinsuffizienz hervorrufen kann. Aus diesem Grund $\varepsilon$ wurde die gleichzeitige Gabe von Kalzium eingeführt. Der hohe Kaliumgehalt des Konservenblutes kann zusammen mit dem beim Schock endogen freiwerdenden Kalium ebenfalls zu Transfusionszwischenfällen führen. Ausserdem kann der niedrige pH 6,58-6,72 der Blutkonserven die Schockazidose fatal verstärken.

Das Blut der Blutbanken wird bei $4-10^{\circ} \mathrm{C}$ gelagert. Der schockierte Patient zeigt eine eingeschränkte Thermoregulation; die Körpertempera-tur fällt bei Transfusion kalten Blutes. Wenn einem schockierten Patien-ten kaltes Blut rasch infundiert wird, kann es zu einer selektiven kardia-len Hypothermie kommen.

Die Hypothermie spielt eine grosse Rolle für das transfusionsbedingte Kreislaufversagen. Ozinsky konnte zeigen, dass in Fallen, bei denen die Temperatur im mittleren Abschnitt des Oesophagus zwischen 34 und $15^{\circ} \mathrm{C}$ betrug, eine ventrikuläre Fibrillation und Extrasystolie auftrat. Die Ver-mutung liegt nahe, dass die Hypothermie den normalen Metabolismus stört und dadurch die anderen oben genannten Faktoren potenziert, die ebenfalls eine Zirkulationsstörung hervorrufen. Die Koronarien werden als Folge dessen mit einem Blut durchströmt, das wenig Kalziumionen, viel Kalium enthält, sauerstoffarm und reich an sauren Valenzen ist. Diese Situation, eingeleitet durch die Hypothermie, führt zur kardialen Kata-strophe.

Aus diesem Grande sollte das Blut vor der Transfusion in grösseren Mengen auf Körpertemperatur angewärmt werden. Da die Hämolyse der Erythrozyten bei $40^{\circ}$ beginnt, darf diese Temperatur nicht überschritten werden.

Eine Methode, das Blut zu erwärmen, besteht darin, den Transfusions-schlauch zu verlängern und das Blut durch diesen schlangenförmigen Schlauch durch ein Wasserbad zu leiten, was oft nur unter Druck möglich ist.

E. Einen neuen Versuch stellt die Verwendung von hochfrequenten Wellen dar. Diese von Freyzs beschriebene Methode erwärmt das Blut durch hochfrequente elektromagnetische Wellen. Besseling verwendete «radiofrequency induction heating».

Bei diesen Methoden ist das Blut nach 3-5 Minuten erwärmt. BeideMethoden haben sich sehr bewährt. F. Friedrich 